\title{
Nonlocal Quantum Effects in Plasmons of Graphene Superlattices
}

\author{
Luis Brey॰ and T. Stauber \\ Materials Science Factory, Instituto de Ciencia de Materiales de Madrid (CSIC), \\ Cantoblanco, 28049 Madrid, Spain \\ L. Martín-Moreno® \\ Instituto de Ciencia de Materiales de Aragón and Departamento de Física de la Materia Condensada, \\ CSIC-Universidad de Zaragoza, E-50009 Zaragoza, Spain \\ G. Gómez-Santos \\ Departamento de Física de la Materia Condensada, Instituto Nicolás Cabrera \\ and Condensed Matter Physics Center (IFIMAC), Universidad Autónoma de Madrid, \\ E-28049 Madrid, Spain
}

(Received 11 December 2019; accepted 4 June 2020; published 23 June 2020)

\begin{abstract}
By using a nonlocal, quantum mechanical response function we study graphene plasmons in a onedimensional superlattice (SL) potential $V_{0} \cos G_{0} x$. The SL introduces a quantum energy scale $E_{G} \sim$ $\hbar v_{F} G_{0}$ associated with electronic subband transitions. At energies lower than $E_{G}$, the plasmon dispersion is highly anisotropic; plasmons propagate perpendicularly to the SL axis, but become damped by electronic transitions along the SL direction. These results question the validity of semiclassical approximations for describing low energy plasmons in periodic structures. At higher energies, the dispersion becomes isotropic and Drude-like with effective Drude weights related to the average of the absolute value of the local chemical potential. Full quantum mechanical treatment of the kinetic energy thus introduces nonlocal effects that delocalize the plasmons in the SL, making the system behave as a metamaterial even near singular points where the charge density vanishes.
\end{abstract}

DOI: 10.1103/PhysRevLett.124.257401

Introduction.-Graphene plasmons [1-3] have attracted much attention due to their long propagation lengths [4], strong confinement [5], and high gate and frequency tunability [6-17]. Plasmons in pristine graphene are well described by the random phase approximation [18-20], and in the long wavelength limit, the dispersion reads

$$
\omega_{p}=\sqrt{\frac{D}{2 \epsilon \epsilon_{0}} q},
$$

where $D$ is the Drude weight, $q$ the wave vector, and $\epsilon$ the dielectric constant of the surrounding medium. Equation (1) can be derived from a hydrodynamic approach and thus holds for all two-dimensional (2D) systems [21]. For graphene and also for parabolic bands [22], $D \rightarrow D_{0}=$ $\left(e^{2} / \hbar^{2}\right)\left(\left|E_{F}\right| / \pi\right)$, thus in Dirac systems the plasmon frequency depends on the carrier density as $n^{1 / 4}$ [20,23-25].

Exciting plasmons by incident radiation is not possible in pristine graphene because plasmons are strongly confined and energy and momentum conservation prevents their coupling. The coupling can be achieved, though, by superimposing a superlattice (SL) on graphene via external or thermal grating [5,26,27], patterning [28-30], or growing graphene on vicinal surfaces [31]. The SL periodicity induces a folding of the plasmon dispersion, and plasmon subbands appear in the SL Brillouin zone. At the center of the Brillouin zone, the second plasmon subband has its origin in the folding of unperturbed plasmonic modes with momentum $\pm G_{0}$, where $G_{0}$ is the SL reciprocal lattice vector. This plasmon, of finite energy, at the center of the Brillouin zone is inside the light cone and can couple to incident light.

Graphene plasmons in a SL are conventionally discussed within a semiclassical (SC) and local approximation that assumes that the system responds solely to local external fields at each point in space [6,32-37]. This approach is equivalent to assuming that the optical conductivity at each point in space is determined by the Fermi energy at that point which in turn is obtained from the local charge density using the Thomas-Fermi approximation, $\sigma(r)=\sigma_{L}\left(E_{F}[n(r)]\right)$. In these calculations, the main effect of the optical conductivity modulation is the localization of plasmons in the regions of smaller conductivity. Recently, it has been estimated by SC approximations that nonlocal effects are important near regions in which the optical conductivity is strongly suppressed [38]. However, those are the regions where SC approximations are expected to break down, making it necessary to develop a fully quantum-mechanical (QM) approach. 
In this Letter, we study the collective excitations of graphene in the presence of a one-dimensional superlattice potential $V(x)=V_{0} \cos G_{0} x$ within the linear random phase approximation that includes nonlocal and quantummechanical effects ( $Q$ nonlocal). Throughout the Letter, we contrast this approach with two widespread approximations: the local quantum mechanical approximation which reduces the full response matrix to a function ( $Q$ local), and the semiclassical, local approximation which introduces a local Fermi energy in the response function (SC local).

Our main results can be summarized by the following points: (i) absence of Klein tunneling for the motion of plasmons; (ii) at low frequencies, plasmons moving along the SL axis are damped by electronic SL subband excitations; (iii) near singular points, nonlocal effects associated with electron kinetic energy delocalizes the plasmons in the SL unit cell and make the system behave as a metamaterial; (iv) plasmonic excitations in modulated graphene can be related to an effective Drude weight even for vanishing charge density.

Hamiltonian.-The massless Dirac Hamiltonian for one valley and one spin projection in the presence of an external potential is given by

$$
H=\hbar v_{F}\left(-i \sigma_{x} \partial_{x}-i \sigma_{y} \partial_{y}\right)+V(x) \mathcal{I},
$$

where $\sigma_{x}$ and $\sigma_{y}$ are the Pauli matrices, $v_{F}$ is the Fermi velocity, and $\mathcal{I}$ is the identity matrix. Unless otherwise stated, results in this Letter will be presented for an exemplary period of $L=2 \pi / G_{0}=600 a$ with $a=2.46 \AA$. This leads to the energy scale $E_{G}=\hbar v_{F} G_{0} \sim 25 \mathrm{meV}$ which is related to the SL interband electronic transitions. For details on the band structure and further discussion, see the Supplemental Material (SM) [39], in particular the inset of Fig. 1(b).

Plasmonic response.-Plasmonic excitations are the response to an infinitesimal external potential with wave number $\boldsymbol{q}$. However, the induced charge density contains not only the incoming mode $\boldsymbol{q}$, but all higher harmonics $\boldsymbol{q}+G$ with $G=n G_{0}$. The SL potential defines a reduced Brillouin zone where the plasmon subbands are defined. The optical conductivity in the $\nu$ direction thus becomes a matrix of the form $\sigma_{G, G^{\prime}}^{\nu \nu}(\boldsymbol{q}, \omega)$, and the following discussion is based on the energy loss obtained from the largest eigenvalues of the dielectric response and their respective eigenvectors. See the SM for how these quantities are computed [39].

Another quantity of interest will be the Drude weight, which is the static limit of the reactive conductivity, and thus related to electronic intraband transitions. The conductivity also has contributions from electronic interband transitions and plasmons are often characterized by the electronic transitions they are composed of [54,55]. In pristine graphene, plasmons are usually Drude-like and have no contributions from the interband conductivity. However, a SL potential creates both low energy electronic

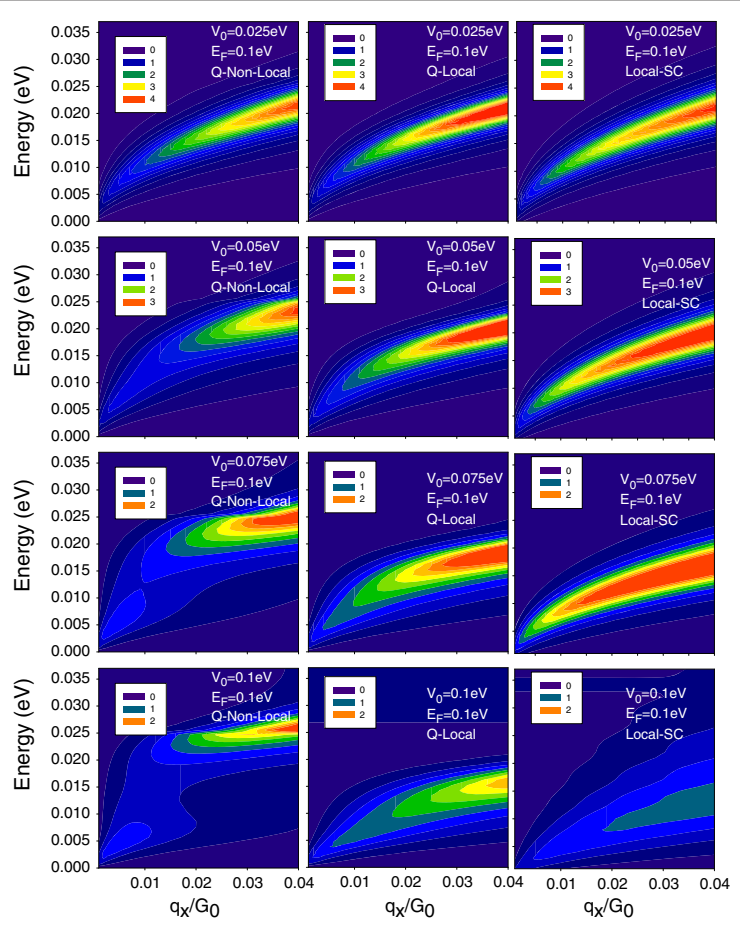

FIG. 1. Intraband plasmon dispersion (energy loss) with (left) and without (middle) nonlocal effects for a superlattice with period $L=600 a$ and different values of $V_{0}$, as obtained in the quantum mechanical calculation. The right panels show the local semiclassical results. The comparison along the $y$ direction is omitted since the Drude weight does not depend on $V_{0}$.

subbands and spectral weight transfer from electronic intraband to electronic interband transitions. This is a QM effect that is not included in SC calculations, in which the electronic subbands of the SL are not taken into account and all the plasmons are intrabandlike.

In the following we will consider two regimes. First, we fix the Fermi energy to $E_{F}=0.1 \mathrm{eV}\left(E_{F} \gg E_{G}\right)$ and then vary the superlattice potential $V_{0}$ from zero (uniform limit) up to $E_{F}$ where the electron density becomes zero at discrete locations, so-called singular points [38]. By this, we analyze how the collective excitations change as the system becomes more and more inhomogeneous. Second, we analyze a system with average zero density $\left(E_{F} \ll E_{G}\right)$ where the SL potential creates alternating $p$ - and $n$-doped regions. In each of these regimes, we will analyze the dispersion of the first plasmonic subband and the plasmonic resonance that appears in the second subband at the center of the Brillouin zone. This zero momentum plasmon can be probed in optical transmittance and reflectance measurements.

Finite $E_{F}$ : First plasmonic subband. Let us first discuss the loss function for collective excitations with momentum $q_{y}$. We observe a well-defined low energy branch that disperses as $\sqrt{\left(D_{0} / 2 \epsilon \epsilon_{0}\right) q_{y}}$ with the same Drude weight as obtained from pristine graphene. This part agrees with the $\mathrm{SC}$ approximation. 
On the contrary, the Drude weight in the direction of the superlattice is reduced for increasing $V_{0}$ due to transfer of spectral weight from interband to intraband transitions [39]. This is also manifested in the loss function which is shown in the left column of Fig. 1 for various amplitudes $V_{0} / E_{F}$. Increasing the modulation $V_{0}$ renders the plasmons ill defined below an energy threshold which we identify as $E_{G}$.

A large dissipative plasmonic response at low energies is also seen in metallic structures with nanogap features $[56,57]$. However, the Q-local and semiclassical ThomasFermi approximations fail to predict this threshold energy $E_{G}$, as shown in the center and right panels of Fig. 1.

The vanishing of the loss function is the result of new decay channels that develop due to electronic band folding that allows for electron-hole transitions at energies $\hbar \omega \lesssim E_{G}$. An equivalent viewpoint is that umklapp processes scatter the plasmonic excitations with $\boldsymbol{q} \sim 0$ into the particle-hole continuum of intraband transitions where they decay due to strong Landau damping. This is only possible for $\hbar \omega \lesssim E_{G}$ and a purely nonlocal and quantum-mechanical effect since plasmonic band folding is only encoded in $\sigma_{G, G}^{\nu \nu}$ with $G \neq 0$. It is thus even absent in the quantum-local approximation $\sigma_{G, G^{\prime}}^{\nu \nu} \rightarrow \sigma_{G-G^{\prime}, 0}^{\nu \nu}$. For energies $E \gtrsim E_{G}$, the loss function becomes isotropic, Figs. 2(a) and 2(b), and displays a well-defined Drude-like dispersion, with a Drude
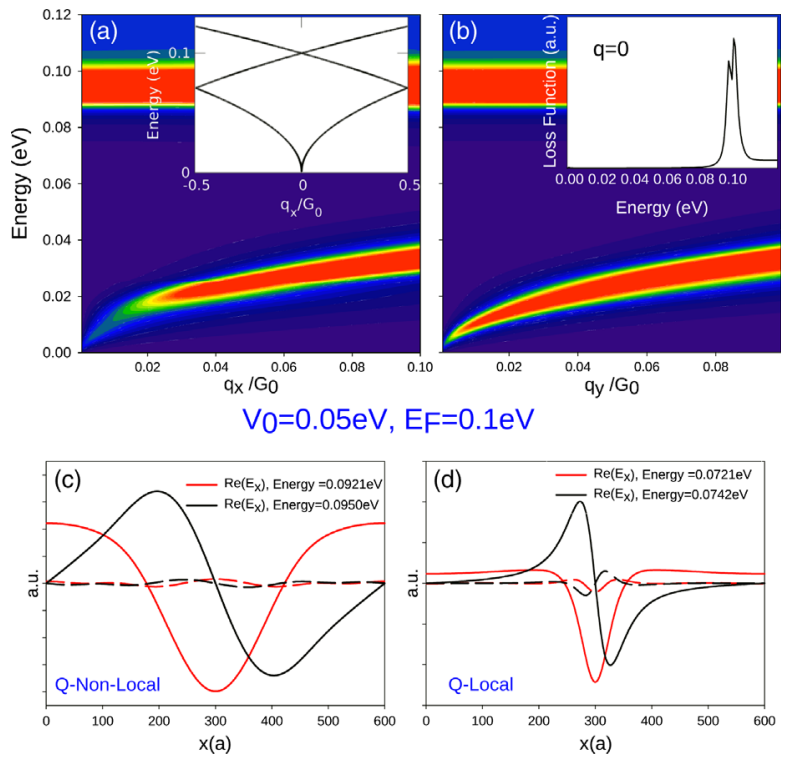

FIG. 2. Quantum nonlocal energy loss as function of (a) $q_{x}$ and (b) $q_{y}$ for a SL of period $L=600 a$, Fermi energy $E_{F}=0.1 \mathrm{eV}$, and amplitude $V_{0}=0.05 \mathrm{eV}$. In the inset of panel (a), we plot the idealized illustration of the folding mechanism for the emergence of the second plasmonic subband. In the inset of panel (b), we plot the energy loss for $\mathbf{q}=0$. Panel (c) shows the real (full lines) and imaginary (dashed lines) part of the electric fields, respectively, of the two strongest subband dielectric eigenmodes at $\mathbf{q}=0$. In panel (d), we plot the same as in panel (c), but for the eigenmodes obtained in the Q-local approximation. weight value equal to the intraband Drude weight in the $y$ direction.

Remarkably, the anisotropy in the plasmon dispersion is opposite to that occurring in the electronic band structure of the SL [39-44], where, due to Klein tunneling the velocity of the electrons moving along the SL axis is not modified, whereas it is strongly reduced in the perpendicular direction. The reason is that plasmons are collective excitations which even if characterized by a wave vector parallel to the superlattice are composed by electrons and holes moving in a range of directions. Plasmons are thus hardly affected by the Klein paradox.

Finite $E_{F}$ : Second plasmonic subband. In Figs. 2(a) and 2(b), we plot the energy loss function at the center of the Brillouin zone for a SL with $V_{0}=E_{F} / 2$. In fact, the loss function presents a double peak structure, see inset of Fig. 2(a), which widens and becomes less intense for increasing $V_{0}$. The double peak structure reflects the folding onto $\mathbf{q}=0$ of two states with momentum $\pm G_{0}$. These states interact between them presenting a small energy splitting proportional to $V_{0}$, as can be seen in the inset of Fig. 2 and also in the SM [39].

The modes in the second plasmon subband have a strong contribution from electronic interband transitions generated by the SL potential. This contribution increases as the SL perturbation $V_{0}$ increases. Nevertheless, the energy of the second plasmonic subband is very close to $\hbar \omega_{p}\left(G_{0}\right)$, i.e., related to the Drude weight evaluated at the Fermi energy. The energy difference with respect to Eq. (1) can be explained by nonlocal effects of the conductivity [18,20], see SM [39].

In Fig. 2(c), we plot the $\hat{x}$ component of the electric field corresponding to the two eigenmodes that appear in the inset of Fig. 2(a). The electric fields have the form of sine and cosine functions and thus correspond to the combination of the folded states with momentum $\pm G_{0}$, showing that the plasmon modes can be obtained from folding of the unperturbed plasmons, even in this case of large amplitude of the perturbation. The length scale of the interband plasmons is given by the period of the superlattice, i.e., $L \sim 1 / G_{0}$, and are thus not confined. In Fig. 2(d), we plot the electric fields corresponding to the double peaks that appear in the Q-local approximation. Again, the two dielectric modes correspond to even and odd functions, but the electric fields are localized in the region of low density as the SC local approximation also predicts [38].

Hence, nonlocal effects allow plasmons to explore all spatial regions and become extended over the whole system, making the SL behave as a metamaterial displaying a homogeneous optical response even in the presence of singular points where the electron density vanishes. This contrasts with the results obtained in local approximations (both SC and quantum) which are especially drastic near singular points.

$E_{F}=0$ : Neutral plasmons. For neutral graphene, we have $D_{0}=0$ at zero temperature, signaling the lack of 
plasmons. However, at finite temperature there is an effective Drude weight and plasmons can be defined [58-60]. In this work, we show that in neutral, but modulated graphene, band folding leads to the appearance of plasmon subbands for $V_{0} \gtrsim E_{G}$. Since for undoped samples, electronhole symmetry with respect to the chemical potential implies that the system responds just to the absolute value of the SL potential, there is a halving of the periodicity of the modulation, shown to be an exact symmetry in the SM [39]. Therefore, the electronic subband energy scale is in this case related to $E_{G} \rightarrow 2 \hbar v_{F} G_{0}$. In the first plasmonic subband, the dispersion shows a strong anisotropy: as in the case of finite doping, we find a plasmonic gap in the $x$ direction, whereas unperturbed intraband plasmons in the $y$ direction, see Fig. 4 in the SM [39].

At the center of the Brillouin zone, we find that for large values of the modulation $V_{0}$, the loss function displays a clear maximum that indicates the existence of well defined plasmonic resonances, see Fig. 8 of the SM [39]. When decreasing $V_{0}$, the energy of the plasmon is redshifted, the peak broadens and a continuous background appears at high energies, eventually signaling the absence of plasmons. In Fig. 3, we plot the energy of this resonance as a function of the folded momentum $2 G_{0}$ in units of the effective Fermi energy $\bar{E}_{F}=(2 / \pi) V_{0}$ and effective Fermi wave vector $\bar{k}_{F}=\left(\bar{E}_{F} / \hbar v_{F}\right)$, respectively. The red dots thus correspond to peak positions for different values of $V_{0}$ and follow the Drude dispersion of pristine graphene of Eq. (1) evaluated at $\bar{E}_{F}$ (blue dashed line).

The same behavior can be seen for different periodicities $L=900 a$ (magenta dots) and $L=300 a$ (green dots) which demonstrates that electronic interband transitions in fact mimic an effective classical Drude weight $D \sim V_{0}$. However, as shown in the inset of Fig. 3, the effective

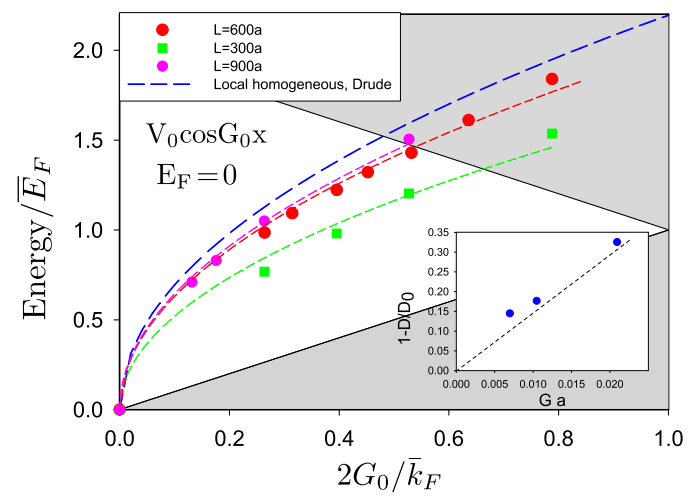

FIG. 3. Second subband plasmonic energies for SL potentials with period $L=900 a$ (magenta), $L=600 a$ (red), and $L=300 a$ (green) for different values of $V_{0} \leq 0.2 \mathrm{eV}$. The grey regions indicate the existence of electron hole damping by intra- or interband (electronic) excitations. The dashed lines are the corresponding fits to Eq. (1) extracting the effective Drude weight $D$. Inset: The effective Drude weight as function of the reciprocal lattice vector. classical Drude weight is redshifted by quantum effects associated with interband transitions and are related to $E_{G}$. For a more detailed discussion, see the SM [39].

We finally note that one-dimensional edge plasmons have been predicted to exist at the interface of a $p-n$ junction which show an unusual $q^{1 / 4}$ dispersion [61]. We do not observe this effect, because their existence requires wavelengths much larger than the width of the junction, while here we have addressed the opposite limit.

Summary of different Drude regimes. - As noted above, the zero momentum finite energy plasmonic excitations can be described by Eq. (1), albeit with an effective wave number $G$ and effective Drude weight $D$. The actual values depend on the ratio between the Fermi energy $E_{F}$, the modulation $V_{0}$, and the quantum electronic subband energy $E_{G}=\hbar v_{F} G$. For $E_{F}>V_{0}$, the wave vector $G$ coincides with the SL reciprocal lattice vector $G_{0}$ whereas for $E_{F} \ll V_{0}$, a periodicity halving occurs [62] and the relevant wave vector is $G=2 G_{0}$.

The different Drude regimes are illustrated in Fig. 4 and defined in the following way:

(i) For $\hbar \omega<E_{G}$, the Drude weight in the $\nu$ direction is given by [63]

$$
D_{\nu}=\lim _{\omega \rightarrow 0} \omega \operatorname{Im} \sigma_{G=0, G=0}^{\nu \nu}(q=0, \omega) .
$$

In the limit $V_{0} \rightarrow E_{F}$, the Drude weight becomes highly anisotropic and the plasmons ill defined in the direction parallel to the SL, $D_{x} \sim 0$.

(ii) For $\hbar \omega>E_{G}$, the isotropic Drude weight is given by

$$
D=\frac{e^{2}}{\hbar^{2}} \frac{\overline{\left|E_{F}+V(x)\right|}}{\pi},
$$

where the average over one unit cell of the superlattice is implied. Moreover, the plasmon dispersion is isotropic, $D \sim D_{x} \sim D_{y}$ and the Drude weight agrees well with Eq. (3) in the $\hat{y}$ direction. For $E_{F} \ll E_{G}$, a redshift $\sim E_{G}$ needs to be included in Eq. (4), see inset of Fig. 3.

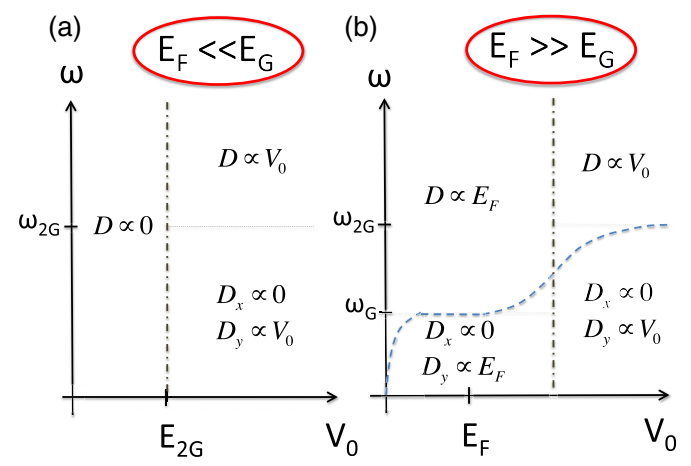

FIG. 4. Schematic map of the Drude weights for (a) $E_{F} \ll E_{G}$ and (b) $E_{F} \gg E_{G}$. All lines indicate crossover behavior. We assume electron doping with $E_{F}>0$ and nonlocal or lattice corrections are not included. 
Conclusions.-We have analyzed the plasmonic properties of graphene dressed by a one-dimensional superlattice potential. Even though the superlattice potential leads to the emergence of multiple electronic subbands, the character of the plasmonic excitations is not changed, i.e., they remain Drude-like charge-density oscillations where only the charge stiffness enters as an effective parameter. In contrast to what both SC and Q-local approximations predict, only minor corrections related to $V_{0}$ are observed when the modulation potential reaches the singular-point regime, $V_{0} \sim E_{F}$. In the case of a neutral SL formed by periodically alternating $n$ - and $p$-doped regions, we observe a halving of the periodicity, indicating that plasmons are insensitive to the sign of its carriers, in agreement with exact results [39]. More interestingly, we again find Drude-like behavior of the subband resonances related to $\omega_{p}\left(2 G_{0}\right)$.

We find that quantum electronic intersubband transitions damp the propagation of plasmon excitations along the SL axis, questioning the use of semiclassical calculations for this low frequency regime. The classical approach is valid whenever the external grating does not induce a sufficiently large modulation of the "local" Fermi energy. In a quantum mechanical treatment, the Fermi energy is constant over the whole sample and the electronic density becomes inhomogeneous, instead. This variation can be controlled by bringing, e.g., the metal gratings closer to the graphene layer in order to enhance the screening effects and thus the local depletion of the electronic density. A rough estimate would be given by $V_{0} / E_{F} \sim 0.1$. Another scale to contrast the classical approach is given by $E_{G}$ which is entirely due to band folding. Thus, reducing the periodicity will eventually lead to deviations and a rough estimate is given by $L \sim 1 \mu \mathrm{m}$. In the case of plasmons with wavelengths of the order of the SL period, nonlocal effects reflected by the wave nature of the electrons delocalize the plasmons in the SL unit cell, making it possible to describe the system as metamaterial.

Finally, let us point out that the collective excitations show an opposite behavior to that of single-particle Dirac excitations, i.e., plasmons are largely unaffected by the superlattice in the direction perpendicular to the modulation, but strongly modified in the parallel direction. Klein tunneling thus has no or very little effect on the plasmon propagation, contrary to several claims in the literature.

This work has been supported by Spain's MINECO under Grants No. PGC2018-097018-B-100, No. PGC2018-096955-B-C42, No. FIS2017-82260-P, No. MAT2017-88358-C3-1-R, and No. MDN-2014-0377 as well as by the CSIC Research Platform on Quantum Technologies PTI-001. L. M.-M. acknowledges the Aragón Government through project Q-MAD.

[1] L. Ju, B. Geng, J. Horng, C. Girit, M. Martin, Z. Hao, H. A. Bechtel, X. Liang, A. Zettl, Y. R. Shen, and F. Wang, Nat. Nanotechnol. 6, 630 (2011).
[2] J. Chen, M. Badioli, P. Alonso-Gonzalez, S. Thongrattanasiri, F. Huth, J. Osmond, M. Spasenovic, A. Centeno, A. Pesquera, P. Godignon, A. Zurutuza Elorza, N. Camara, F. J. G. de Abajo, R. Hillenbrand, and F. H. L. Koppens, Nature (London) 487, 77 (2012).

[3] Z. Fei, A. S. Rodin, G. O. Andreev, W. Bao, A. S. McLeod, M. Wagner, L. M. Zhang, Z. Zhao, M. Thiemens, G. Dominguez, M. M. Fogler, A. H. C. Neto, C. N. Lau, F. Keilmann, and D. N. Basov, Nature (London) 487, 82 (2012).

[4] G. X. Ni, A. S. McLeod, Z. Sun, L. Wang, L. Xiong, K. W. Post, S. S. Sunku, B. Y. Jiang, J. Hone, C. R. Dean, M. M. Fogler, and D. N. Basov, Nature (London) 557, 530 (2018).

[5] D. Alcaraz Iranzo, S. Nanot, E. J. C. Dias, I. Epstein, C. Peng, D. K. Efetov, M. B. Lundeberg, R. Parret, J. Osmond, J.-Y. Hong, J. Kong, D. R. Englund, N. M. R. Peres, and F. H. L. Koppens, Science 360, 291 (2018).

[6] A. Nikitin, Graphene Plasmonics, World Scientific Handbook of Metamateriasl and Plasmonics, Vol. 1 (Word Scientific Publishing, Singapore, 2017).

[7] F. H. L. Koppens, D. E. Chang, and F. J. García de Abajo, Nano Lett. 11, 3370 (2011).

[8] T. Low, A. Chaves, J. D. Caldwell, A. Kumar, N. X. Fang, P. Avouris, T. F. Heinz, F. Guinea, L. Martin-Moreno, and F. Koppens, Nat. Mater. 16, 182 (2016).

[9] Z. Torbatian and R. Asgari, Appl. Sci. 8, 238 (2018).

[10] F. J. García de Abajo, ACS Photonics 1, 135 (2014).

[11] P. Goncalves and N. Peres, An Introduction to Graphene Plasmonics (World Scientific, Singapore, 2016).

[12] F. Bonaccorso, Z. Sun, T. Hasan, and A. C. Ferrari, Nat. Photonics 4, 611 (2010).

[13] D. B. Farmer, D. Rodrigo, T. Low, and P. Avouris, Nano Lett. 15, 2582 (2015).

[14] A. I. Fernández-Domínguez, F. J. García-Vidal, and L. Martín-Moreno, Nat. Photonics 11, 8 (2017).

[15] E. Galiffi, J. B. Pendry, and P. A. Huidobro, ACS Nano 12, 1006 (2018).

[16] Y. Fan, N.-H. Shen, F. Zhang, Q. Zhao, H. Wu, Q. Fu, Z. Wei, H. Li, and C. M. Soukoulis, Adv. Opt. Mater. 7, 1800537 (2019).

[17] B. Foerster, V. A. Spata, E. A. Carter, C. Sönnichsen, and S. Link, Sci. Adv. 5, eaav0704 (2019).

[18] B. Wunsch, T. Stauber, F. Sols, and F. Guinea, New J. Phys. 8, 318 (2006).

[19] T. Ando, J. Phys. Soc. Jpn. 75, 074716 (2006).

[20] E. H. Hwang and S. Das Sarma, Phys. Rev. B 75, 205418 (2007).

[21] T. Ando, A. B. Fowler, and F. Stern, Rev. Mod. Phys. 54, 437 (1982).

[22] T. Stauber, J. Phys. Condens. Matter 26, 123201 (2014).

[23] L. Brey and H. A. Fertig, Phys. Rev. B 75, 125434 (2007).

[24] J. Horng, C.-F. Chen, B. Geng, C. Girit, Y. Zhang, Z. Hao, H. A. Bechtel, M. Martin, A. Zettl, M. F. Crommie, Y. R. Shen, and F. Wang, Phys. Rev. B 83, 165113 (2011).

[25] H. Yoon, C. Forsythe, L. Wang, N. Tombros, K. Watanabe, T. Taniguchi, J. Hone, P. Kim, and D. Ham, Nat. Nanotechnol. 9, 594 (2014).

[26] N. M. R. Peres, Y. V. Bludov, A. Ferreira, and M. I. Vasilevskiy, J. Phys. Condens. Matter 25, 125303 (2013). 
[27] R. Yu, Q. Guo, F. Xia, and F. J. García de Abajo, Phys. Rev. Lett. 121, 057404 (2018).

[28] L. Ju, B. Geng, J. Horng, C. Girit, M. Martin, Z. Hao, H. A. Bechtel, X. Liang, A. Zettl, Y. R. Shen, and F. Wang, Nat. Nanotechnol. 6, 630 (2011).

[29] M. Drienovsky, J. Joachimsmeyer, A. Sandner, M.-H. Liu, T. Taniguchi, K. Watanabe, K. Richter, D. Weiss, and J. Eroms, Phys. Rev. Lett. 121, 026806 (2018).

[30] L. Xiong, C. Forsythe, M. Jung, A. S. McLeod, S. S. Sunku, Y. M. Shao, G. X. Ni, A. J. Sternbach, S. Liu, J. H. Edgar, E. J. Mele, M. M. Fogler, G. Shvets, C. R. Dean, and D. N. Basov, Nat. Commun. 10, 4780 (2019).

[31] A. Celis, M. N. Nair, M. Sicot, F. Nicolas, S. Kubsky, D. Malterre, A. Taleb-Ibrahimi, and A. Tejeda, Phys. Rev. B 97, 195410 (2018).

[32] N. M. R. Peres, A. Ferreira, Y. V. Bludov, and M. I. Vasilevskiy, J. Phys. Condens. Matter 24, 245303 (2012).

[33] T. M. Slipchenko, M. L. Nesterov, L. Martin-Moreno, and A. Y. Nikitin, J. Opt. 15, 114008 (2013).

[34] I. Silveiro, A. Manjavacas, S. Thongrattanasiri, and F. J. García de Abajo, New J. Phys. 15, 033042 (2013).

[35] C. Beckerleg and E. Hendry, J. Opt. Soc. Am. B 33, 2051 (2016).

[36] P. A. Huidobro, M. Kraft, S. A. Maier, and J. B. Pendry, ACS Nano 10, 5499 (2016).

[37] P. A. Huidobro, M. Kraft, R. Kun, S. A. Maier, and J. B. Pendry, J. Opt. 18, 044024 (2016).

[38] E. Galiffi, P. A. Huidobro, P. A. D. Goncalves, N. A. Mortensen, and J. B. Pendry, Nanophotonics 9, 309 (2020).

[39] See Supplemental Material at http://link.aps.org/ supplemental/10.1103/PhysRevLett.124.257401 for more details and additional analytical and numerical results, which includes the additional Refs. [40-53].

[40] C.-H. Park, L. Yang, Y.-W. Son, M. L. Cohen, and S. G. Louie, Nat. Phys. 4, 213 (2008).

[41] M. Barbier, F. M. Peeters, P. Vasilopoulos, and J. M. Pereira, Phys. Rev. B 77, 115446 (2008).

[42] D. P. Arovas, L. Brey, H. A. Fertig, E.-A. Kim, and K. Ziegler, New J. Phys. 12, 123020 (2010).

[43] P. Burset, A. L. Yeyati, L. Brey, and H. A. Fertig, Phys. Rev. B 83, 195434 (2011).
[44] L. Brey and H. A. Fertig, Phys. Rev. Lett. 103, 046809 (2009).

[45] C.-H. Park, Y.-W. Son, L. Yang, M. L. Cohen, and S. G. Louie, Phys. Rev. Lett. 103, 046808 (2009).

[46] T. Stauber, G. Gómez-Santos, and L. Brey, ACS Photonics 4, 2978 (2017).

[47] G. Giuliani and G. Vignale, Quantum Theory of the Electron Liquid (Cambridge University Press, Cambridge, 2005).

[48] A.Baldereschi and E.Tosatti, Solid State Commun. 29, 131 (1978).

[49] R. Car, E. Tosatti, S. Baroni, and S. Leelaprute, Phys. Rev. B 24, 985 (1981).

[50] K. Andersen, K. W. Jacobsen, and K. S. Thygesen, Phys. Rev. B 86, 245129 (2012).

[51] W. Wang, T. Christensen, A.-P. Jauho, K. S. Thygesen, M. Wubs, and N.A. Mortensen, Sci. Rep. 5, 9535 (2015).

[52] J. Sabio, J. Nilsson, and A. H. Castro Neto, Phys. Rev. B 78, 075410 (2008).

[53] J. H. Strait, P. Nene, W.-M. Chan, C. Manolatou, S. Tiwari, F. Rana, J. W. Kevek, and P. L. McEuen, Phys. Rev. B 87, 241410(R) (2013).

[54] L. A. Falkovsky and S. S. Pershoguba, Phys. Rev. B 76, 153410 (2007).

[55] T. Stauber, N. M. R. Peres, and A. K. Geim, Phys. Rev. B 78, 085432 (2008).

[56] W. Yan, M. Wubs, and N. Asger Mortensen, Phys. Rev. Lett. 115, 137403 (2015).

[57] W. Zhu, R. Esteban, A. G. Borisov, J. J. Baumberg, P. Nordlander, H. J. Lezec, J. Aizpurua, and K. B. Crozier, Nat. Commun. 7, 11495 (2016).

[58] O. Vafek, Phys. Rev. Lett. 97, 266406 (2006).

[59] G. Gómez-Santos, Phys. Rev. B 80, 245424 (2009).

[60] T. Stauber and G. Gómez-Santos, New J. Phys. 14, 105018 (2012).

[61] E. G. Mishchenko, A. V. Shytov, and P. G. Silvestrov, Phys. Rev. Lett. 104, 156806 (2010).

[62] H. Wang, D. Nezich, J. Kong, and T. Palacios, IEEE Electron Device Lett. 30, 547 (2009).

[63] T. Stauber, P. San-Jose, and L. Brey, New J. Phys. 15, 113050 (2013). 